\title{
Elevated DKKI expression is an independent unfavorable prognostic indicator of survival in head and neck squamous cell carcinoma
}

This article was published in the following Dove Press journal:

Cancer Management and Research

\author{
Haihe Gao' \\ Lisha $\mathrm{Li}^{2}$ \\ Mang Xiao ${ }^{3}$ \\ Yongwei Guo ${ }^{4}$ \\ Yi Shen ${ }^{5,6}$ \\ Lixin Cheng ${ }^{5}$ \\ Ming Tang 5 \\ 'Department of Otolaryngology \\ Head and Neck Surgery, Huashan \\ Hospital, Fudan University, Shanghai \\ 200040, People's Republic of China; \\ ${ }^{2}$ Department of Nursing, Ningbo \\ Medical Treatment Center Lihuili \\ Hospital, Ningbo 31504I, People's \\ Republic of China; ${ }^{3}$ Department \\ of Otolaryngology Head and \\ Neck Surgery, Sir Run Run Shaw \\ Hospital, Zhejiang University School \\ of Medicine, Hangzhou, People's \\ Republic of China; ${ }^{4}$ Department \\ of Ophthalmology, University of \\ Cologne, 50937, Cologne, Germany; \\ ${ }^{5}$ Department of Otorhinolaryngology \\ Head and Neck Surgery, Ningbo \\ Medical Center Lihuili Hospital, \\ Ningbo 3 I 5040, People's Republic \\ of China; ${ }^{6}$ Department of \\ Otolaryngology Head and Neck \\ Surgery, School of Medicine, Ningbo \\ University, Ningbo 3152II, People's \\ Republic of China
}

Correspondence: Ming Tang Department of Otorhinolaryngology Head and Neck Surgery, Ningbo Medical Center Lihuili Hospital, 57 Xingning Road, Ningbo, 315040 Zhejiang, People's Republic of China

Tel +86 I33 45959249

Fax +86 57487392232

Email tmtm-50@163.com
Purpose: DKK1 is an antagonist of the Wnt signaling pathway that has various roles in human physiology. Notably, aberrant $D K K 1$ expression is observed in several cancers. In this retrospective study, we assessed the association between $D K K 1$ expression levels and head and neck squamous cell carcinoma (HNSCC) and its prognostic value.

Materials and methods: Using RNA-seq data from HNSCC tumors $(\mathrm{N}=520)$ and adjacent normal tissue $(\mathrm{N}=44)$ in The Cancer Genome Atlas, we evaluated DKK1 expression levels. Additionally, we evaluated the association of $D K K 1$ expression levels and pathophysiological features of patients with HNSCC and the value of $D K K 1$ expression for prediction of overall survival (OS). We also explored the correlation between $D K K 1$ expression and methylation of its promoter in HNSCC.

Results: $D K K 1$ expression was significantly upregulated in HNSCC compared with normal tissues. Moreover, $D K K 1$ expression was significantly associated with smoking, alcohol abuse, sex, human papillomavirus status, tumor site, tumor invasion, and pathologic stage in HNSCC patients. Kaplan-Meier curves showed that high $D K K 1$ expression was correlated with inferior OS. In addition, univariate and multivariate analyses showed that elevated $D K K 1$ expression was an independent prognostic factor for poor OS (HR: $1.85,95 \% \mathrm{CI}: 1.31-2.62, P<0.001$ ). Regression analysis identified a strong negative correlation between $D K K 1$ expression and methylation of its promoter.

Conclusion: These findings support the hypothesis that elevated $D K K 1$ expression is modulated via methylation of its promoter and indicate that $D K K 1$ expression is a highly informative prognostic biomarker for patients with HNSCC.

Keywords: HNSCC, DKK1, overall survival, prognosis, methylation

\section{Introduction}

Head and neck cancer is the sixth most common malignancy and fifth leading cause of cancer-related death worldwide, and mainly comprises head and neck cancer squamous cell carcinomas (HNSCCs) arising from the mucosal surfaces of the upper-aerodigestive tract (oral and nasal cavity, oropharynx, hypopharynx, and larynx). ${ }^{1}$ According to recent epidemiological data from the American Cancer Society, HNSCC incidence and mortality rates have both increased in recent years. ${ }^{2,3}$ Despite aggressive multimodal treatment approaches, including surgical resection, chemotherapy, targeted therapy, and radiotherapy, the 5-year survival rate of patients with HNSCC is $<50 \%,{ }^{4}$ because the majority are diagnosed with advanced-stage disease $^{5}$ and there is a high risk of local recurrence and/or distant metastatic treatment failure. ${ }^{6}$ Therefore, identification of potential markers to predict prognosis or 
optimize therapy is a matter of clinical urgency for patients with HNSCC.

DKK1, a member of the DKK family, is an antagonist of Wnt signaling, which regulates diverse cellular and biological processes, including cell proliferation, migration, and apoptosis, through both $\beta$-catenin-dependent and -independent mechanisms, in various tissues and numerous cancers. ${ }^{7-9}$ Abnormal expression of $D K K 1$ is observed in numerous tumors and contributes to malignant transformation. In human renal cell carcinoma, $D K K 1$ acts as a tumor suppressor gene that induces apoptosis and inhibits proliferation. ${ }^{10}$ Pang et $\mathrm{al}^{11}$ demonstrated that upregulation of $D K K 1$ in SBC-3 cells enhanced their proliferation, colony formation, cell migration, and invasion in vitro, as well as bone metastasis in vivo. In prostate cancer, levels of $D K K 1$ were found to increase during cancer development and were associated with inferior patient survival. ${ }^{12}$ A recent study showed that $D K K 1$ expression is also elevated in the Hep-2 cell line and laryngeal squamous cell carcinoma samples and has potential as an independent unfavorable predictor of overall survival (OS) in patients with this type of cancer; ${ }^{13}$ however, the small sample size limited the statistical power of the study and the association of $D K K 1$ with HNSCC remains insufficiently investigated.

The Cancer Genome Atlas (TCGA), supervised by the National Cancer Institute's Center for Cancer Genomics and the National Human Genome Research Institute, is an open data-rich resource for biological discovery that has collated information from more than 10,000 patient samples, together with clinicopathological data, across over 30 types of human cancers. ${ }^{14}$ Genomic and phenotypic data from $528 \mathrm{HNSCC}$ and normal tissues are recorded in the HNSCC-specific section of the database, TCGA-HNSC. In this retrospective study, using data from TCGA-HNSC, we explored the DKK1 expression levels in HNSCC, their association with disease clinicopathological characteristics, and their prognostic value.

DNA methylation is a common epigenetic modification, and aberrant promoter methylation at $\mathrm{CpG}$ islands in gene promoters leads to transcriptional inactivation, which is critical in cancer initiation, progression, invasion, and metastasis. ${ }^{15-17}$ Accumulating evidence demonstrates that transcriptional silencing of $D K K 1$ by methylation of its promoter is observed in hepatocellular carcinoma, ${ }^{18}$ nonsmall-cell lung cancer, ${ }^{19}$ colorectal cancer, ${ }^{20}$ and oral and oropharyngeal cancer. ${ }^{21}$ In the current study, we also explored the association between $D K K 1$ expression and methylation of its promoter using Illumina Human Methylation 450K
BeadChip data (Illumina Inc., San Diego, CA, USA) from TCGA-HNSC.

\section{Materials and methods Available TCGA data}

TCGA-HNSCC cohort data (Project Id: TCGA-HNSC) were downloaded from the University of California Santa Cruz Xena browser (https://xenabrowser.net/). Gene expression data, measured by RNA-seq, were available for 520 primary HNSCC tumor and 44 adjacent normal tissue samples; 517 primary HNSCC cases had both RNA-seq and OS data recorded. Details of the clinicopathological characteristics of patients, including age at initial pathologic diagnosis, sex, smoking history, alcohol history, histologic grade, tumor site, human papillomavirus (HPV) status, pathologic tumor category, pathologic nodal category, nodal pathologic stage, margin status, recurrence status, and OS data, were downloaded for secondary analysis. DNA methylation profiles (Illumina Human Methylation 450K BeadChip Kit) were also downloaded to explore the potential mechanisms of DKK1 dysregulation in HNSCC.

\section{Statistical analyses}

Statistical analyses were performed using SPSS version 20.0 software (IBM Corporation, Armonk, NY, USA) and GraphPad Prism 6.0 (GraphPad Software, Inc., La Jolla, CA, USA). Comparisons between groups were conducted using independent Student's $t$-tests and one-way analysis of variance. For dichotomization of $D K K 1$ expression values, the 517 primary HNSCC patients with integrated OS data were divided into high and low $D K K 1$ expression groups, according to median gene expression. Survival analyses were conducted by Kaplan-Meier and Cox proportional hazard regression. The average of the $\beta$ values (methylation ratios) of seven Illumina Human Methylation 450K BeadChip probes (cg25454948, cg27411220, cg04932230, cg11931116, cg09445939, cg07684796, and $\operatorname{cg} 27591349)$ on the $D K K 1$ promoter region was calculated and used for subsequent analysis. Correlation between $D K K 1$ expression and methylation was tested using the Spearman's rank correlation coefficient. $P$-values lower than 0.05 were considered significant.

\section{Results}

\section{High DKKI expression levels in HNSCC tumor samples}

In the current study, DKK1 RNA-seq data from 520 patients with histologically confirmed HNSCC and 44 adjacent normal tissues were available from TCGA database. Comparison of DKK1 expression, as determined by RNA-seq, 
demonstrated that $D K K 1$ levels were significantly elevated in $H N S C C$ tissues compared with the adjacent normal samples $(P<0.001$; Figure 1).

\section{Association of DKKI expression with clinicopathological parameters}

We also explored the association of $D K K 1$ expression with the clinicopathological parameters in patients with HNSCC (Table 1). Significantly elevated $D K K 1$ expression levels were found in patients with histories of smoking $(P=0.020)$ and alcohol consumption $(P=0.024)$ in the TCGA-HNSCC cohort. We also identified a difference in DKK1 expression between patients who were HPV- and those who were $\mathrm{HPV}+$, with the latter group having significantly lower $D K K 1$ expression levels $(P<0.001)$. Additionally, we found that hypopharynx and larynx cancers presented with significantly higher DKK1 expression levels compared with oral cavity and oropharynx cancers $(P=0.045)$. Advanced tumor $(\mathrm{T})$ category $(P=0.006)$ and pathologic stage $(P=0.048)$ were also associated with significantly higher $D K K 1$ expression levels compared with early-stage disease. No differences in $D K K 1$ expression were observed according to sex, age, histologic grade, nodal (N) category, surgical margin status, or recurrence status.

\section{High DKKI expression level as an independent prognostic factor for unfavorable OS}

We constructed survival curves to investigate whether $D K K 1$ expression can act as a prognostic biomarker for HNSCC
(Figure 2). The median OS for both the DKK1 high and $D K K 1$ low expression groups were 32.83 and 70.67 months, respectively. Kaplan-Meier analysis and log-rank testing demonstrated that the group with high $D K K 1$ expression had significantly inferior outcome (log-rank $P<0.001)$.

In univariate Cox proportional hazards analysis (Table 2), we found that age $\geq 60$ years, female, advanced $T$, lymphatic metastasis, advanced stage, positive surgical margin, and increased DKK1 expression were significantly associated with inferior OS of patients with HNSCC. To avoid overfitting of the results, univariate Cox regression analysis was also performed using $D K K 1$ expression as a continuous variable. The result confirmed that there was a significantly increased risk of death for patients with high $D K K 1$ expression. Multivariate Cox proportional hazard analysis was conducted including only variables that showed significance in univariate analysis (Table 2). The results confirmed that advanced T, lymphatic metastasis, positive surgical margin, and increased $D K K 1$ expression were independent prognostic indicators of unfavorable OS for patients with HNSCC.

\section{DKKI expression was negatively correlated with methylation of its promoter in HNSCC}

We examined the association of $D K K 1$ expression with methylation of its promoter using data from 520 patients with HNSCC from TCGA. Regression analysis revealed a strong negative correlation (Pearson's $r=-0.515, P<0.001$ between $D K K 1$ expression and methylation of its promoter) (Figure 3).
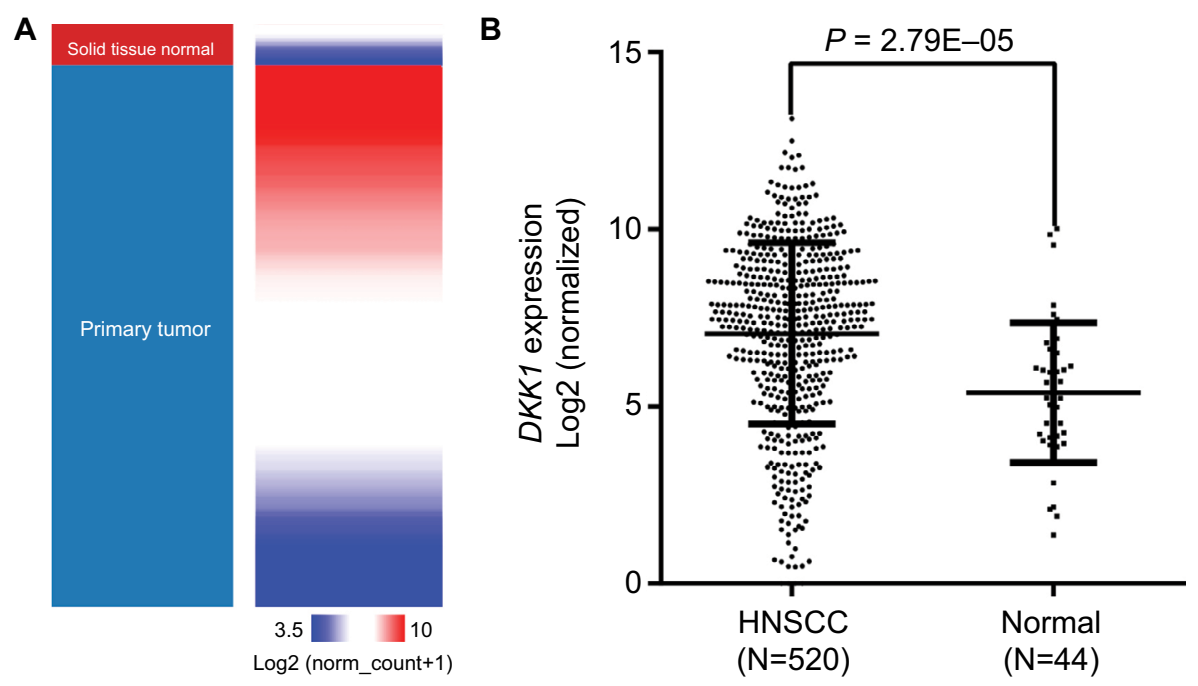

Figure I HNSCC tissues have significantly elevated DKKI expression levels compared with adjacent normal tissues. Note: Heatmap (A) and plot (B) showing DKKI expression in HNSCC tissue ( $\mathrm{T}=520)$ and normal tissue $(\mathrm{N}=44)$. Abbreviation: HNSCC, head and neck squamous cell carcinoma. 
Table I Association of $D K K /$ expression with clinicopathological characteristics of patients with HNSCC

\begin{tabular}{|c|c|c|c|}
\hline Characteristics & $\mathbf{N}$ & Mean \pm SD & $P$-value \\
\hline \multicolumn{4}{|l|}{ Sex } \\
\hline Female & 136 & $7.01 \pm 2.55$ & 0.772 \\
\hline Male & 384 & $7.08 \pm 2.57$ & \\
\hline \multicolumn{4}{|l|}{ Age (years) } \\
\hline$<60$ & 233 & $6.88 \pm 2.67$ & 0.137 \\
\hline$\geq 60$ & 286 & $7.21 \pm 2.47$ & \\
\hline \multicolumn{4}{|l|}{ Smoking behavior } \\
\hline No & 117 & $6.58 \pm 2.67$ & 0.020 \\
\hline Yes & 391 & $7.21 \pm 2.50$ & \\
\hline \multicolumn{4}{|l|}{ Alcohol history } \\
\hline No & 162 & $6.70 \pm 2.6 \mathrm{I}$ & 0.024 \\
\hline Yes & 347 & $7.25 \pm 2.51$ & \\
\hline \multicolumn{4}{|l|}{ Histologic grade } \\
\hline $\mathrm{GI}+2$ & 366 & $7.08 \pm 2.42$ & 0.756 \\
\hline $\mathrm{G} 3+4$ & 132 & $7.16 \pm 2.88$ & \\
\hline \multicolumn{4}{|l|}{ Tumor site } \\
\hline Oral cavity & 385 & $6.88 \pm 2.61$ & 0.045 \\
\hline Oropharynx & 9 & $7.08 \pm 1.30$ & \\
\hline Hypopharynx & 10 & $8.05 \pm 2.06$ & \\
\hline Larynx & 116 & $7.57 \pm 2.45$ & \\
\hline \multicolumn{4}{|l|}{ HPV status } \\
\hline Positive & 41 & $5.12 \pm 2.43$ & $3.66 \times 10^{-5}$ \\
\hline Negative & 73 & $7.21 \pm 2.44$ & \\
\hline \multicolumn{4}{|l|}{$\begin{array}{l}\text { Pathologic tumor } \\
\text { category }\end{array}$} \\
\hline $\begin{array}{l}\text { category } \\
\text { Tis/TI/T2 }\end{array}$ & 185 & $6.78 \pm 2.45$ & 0.006 \\
\hline T3/T4 & 273 & $7.45 \pm 2.59$ & \\
\hline \multicolumn{4}{|l|}{$\begin{array}{l}\text { Pathologic nodal } \\
\text { category }\end{array}$} \\
\hline No & 176 & $7.00 \pm 2.46$ & 0.197 \\
\hline Yes & 244 & $7.33 \pm 2.60$ & \\
\hline \multicolumn{4}{|l|}{ Pathologic stage } \\
\hline$I+I I$ & 101 & $6.76 \pm 2.42$ & 0.048 \\
\hline III + IV & 347 & $7.33 \pm 2.59$ & \\
\hline \multicolumn{4}{|l|}{ Margin } \\
\hline No & 351 & $7.16 \pm 2.58$ & 0.724 \\
\hline Yes & 59 & $7.29 \pm 2.48$ & \\
\hline \multicolumn{4}{|l|}{ Recurrence status } \\
\hline No & 329 & $6.93 \pm 2.60$ & 0.246 \\
\hline Yes & 108 & $7.27 \pm 2.71$ & \\
\hline
\end{tabular}

Abbreviations: HNSCC, head and neck squamous cell carcinoma; HPV human papillomavirus.

\section{Discussion}

The Wnt signaling pathway is a well-conserved and critical regulatory cascade that contributes to the development and homeostasis of the human body. DKK1 is a canonical Wnt signaling pathway inhibitor that has various functions in numerous human diseases, particularly cancers. ${ }^{22-24} \mathrm{~A}$ previous study showed that $D K K 1$ expression is elevated in laryngeal squamous cell carcinoma, which is the second most common malignant HNSCC neoplasm; ${ }^{13}$ however, the relationship between $D K K 1$ and HNSCC remains unclear. In the current study, through characterization of $D K K 1$ expression based on data from TCGA-HNSC, we confirmed that $D K K 1$ expression is significantly upregulated in HNSCC.

Subsequently, we also determined the association between $D K K 1$ expression and the clinicopathological characteristics of patients with HNSCC. The etiologic risk of developing HNSCC is associated with multiple factors, including geographical region, dietary habits, and genetic background, with cigarette smoking and abusive consumption of alcohol representing the most important risk factors for HNSCC development and exhibiting a synergistic effect. ${ }^{25} \mathrm{By}$ analysis of TCGA data, we found that $D K K 1$ expression was significantly elevated in HNSCC patients with smoking and alcohol abuse, suggesting that smoking and alcohol consumption may be involved in the progression of HNSCC via regulation of DKK1. Over the past decade, clear molecular and epidemiologic evidence has emerged supporting a vital role for HPV in a subset of HNSCC tumors, particularly oral and oropharynx squamous cell carcinomas. ${ }^{26,27}$ Also, consistent with prior reports, our analysis showed that $D K K 1$ expression significantly decreased in the HPV associated tumors in TCGA-HNSCC, which may be due to the fact that integration of HPV into the genome results in altered mRNA transcript abundance and splicing. ${ }^{28}$ Tumor invasion and TNM stage are vital factors for assessment of prognosis in patients with cancer and are among the most common tools used for that purpose. ${ }^{29-31}$ Our analyses revealed that $D K K 1$ expression was higher in HNSCC patients with advanced tumor and clinical stage compared with those with early-stage disease, supporting a possible role for $D K K 1$ in the development of HNSCC.

HNSCC is a common type of cancer with a dismal prognosis, and the rates of local or distant recurrence are up to $30 \%$ and $25 \%$, respectively. ${ }^{32}$ TNM staging remains a vital tool for predicting cancer prognosis; $; 3,34$ however, the latest edition of the TNM classification is unable to absolutely justify clinical application, due to the heterogeneous molecular mechanisms and clinical behaviors of HNSCC. Consequently, reliable prognostic biomarkers are urgently needed to aid the risk stratification of patients and inform personalized clinical treatment decision-making processes and subsequent surveillance for HNSCC.

$D K K 1$ upregulation has prognostic value in various cancers, including chondrosarcoma, ${ }^{35}$ bladder cancer, ${ }^{36}$ pancreatic cancer, ${ }^{37}$ and laryngeal squamous cell carcinoma. ${ }^{13}$ In this study, using the large TCGA dataset, Kaplan-Meier 


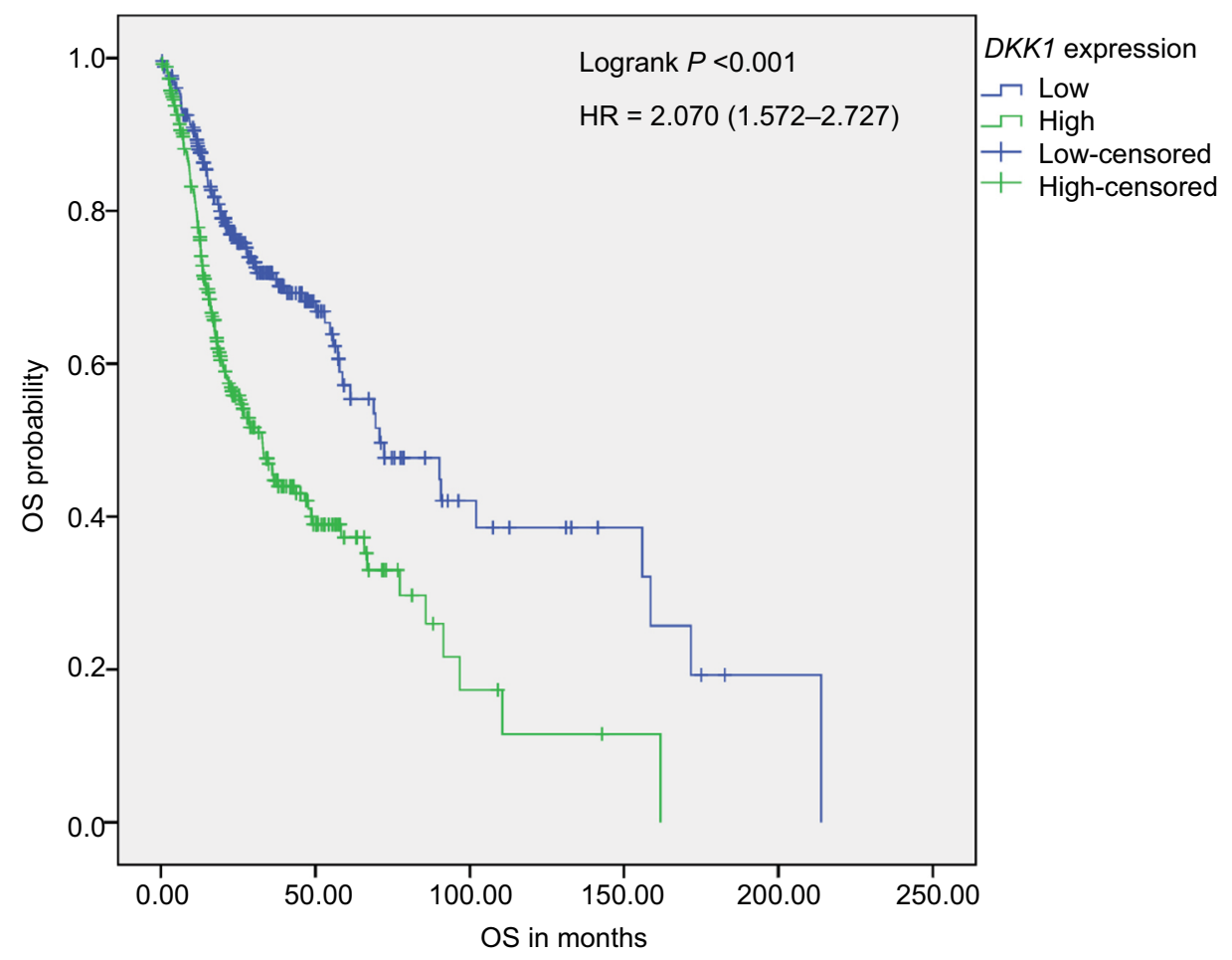

Figure 2 Kaplan-Meier survival analysis.

Notes: Kaplan-Meier analysis of OS in 517 HNSCC patients stratified according to median DKKI expression. OS in patients with high DKKI expression was significantly reduced compared with that of patients with low DKKI expression.

Abbreviations: HNSCC, head and neck squamous cell carcinoma; OS, overall survival.

Table 2 Univariate and multivariate analyses of overall survival in HNSCC patients

\begin{tabular}{|c|c|c|c|c|c|c|}
\hline \multirow[t]{2}{*}{ Characteristics } & \multicolumn{3}{|c|}{ Univariate analysis } & \multicolumn{3}{|c|}{ Multivariate analysis } \\
\hline & HR & $95 \% \mathrm{Cl}$ & $P$-value & HR & $95 \% \mathrm{Cl}$ & $P$-value \\
\hline Age ( $\geq 60$ vs $<60$ years) & 1.32 & $1.00-1.73$ & 0.047 & 1.16 & $0.831-1.63$ & 0.377 \\
\hline Sex (male vs female) & 1.35 & $1.0 \mathrm{I}-1.80$ & 0.040 & 1.43 & $0.979-2.08$ & 0.640 \\
\hline Smoking history (yes vs no) & 1.12 & $0.803-1.57$ & 0.498 & & & \\
\hline Alcohol history (yes vs no) & 0.942 & $0.709-1.25$ & 0.680 & & & \\
\hline Histologic grade (G3/4 vs GI/2) & 0.867 & $0.637-1.18$ & 0.419 & & & \\
\hline Pathologic $\mathrm{T}$ (T3/4 vs TI/2) & 1.91 & $1.40-2.61$ & $<0.001$ & 1.89 & $1.12-3.19$ & 0.017 \\
\hline Pathologic $\mathrm{N}(\mathrm{NI} / 2 / 3$ vs $\mathrm{N} 0)$ & 1.86 & $1.34-2.58$ & $<0.001$ & 1.59 & $1.05-2.42$ & 0.029 \\
\hline Pathologic stage (III/IV vs I/II) & 1.75 & $1.20-2.56$ & 0.004 & 1.14 & $0.512-2.55$ & 0.746 \\
\hline Surgical margin (positive vs negative) & 1.74 & $1.22-2.49$ & 0.002 & 1.71 & I. $14-2.57$ & 0.010 \\
\hline$D K K I$ expression (continuous) & 1.14 & $1.08-1.21$ & $<0.001$ & & & \\
\hline$D K K I$ expression (dichotomized) & 2.07 & $1.57-2.73$ & $<0.001$ & 1.85 & $1.31-2.62$ & $<0.001$ \\
\hline
\end{tabular}

Abbreviations: HNSCC, head and neck squamous cell carcinoma.

survival analysis demonstrated that patients with high $D K K 1$ expression had remarkably shorter OS. In addition, our univariate and multivariate analyses showed that elevated $D K K 1$ expression was an independent prognostic factor for poor OS (HR: 1.85, 95\% CI: 1.31-2.62, $P<0.001$ ), after adjustment for age, sex, tumor invasion, lymphatic metastasis, pathologic stage, and surgical margin status. Therefore, we infer that
$D K K 1$ expression is a potential biomarker of unfavorable OS in HNSCC patients and that implementation of DKK1 expression screening into prospective clinical trials would be highly desirable to provide further support for these findings.

Development of HNSCC is a multistep process that includes various molecular alterations during carcinogenesis. Both genetic and epigenetic alterations can influence 


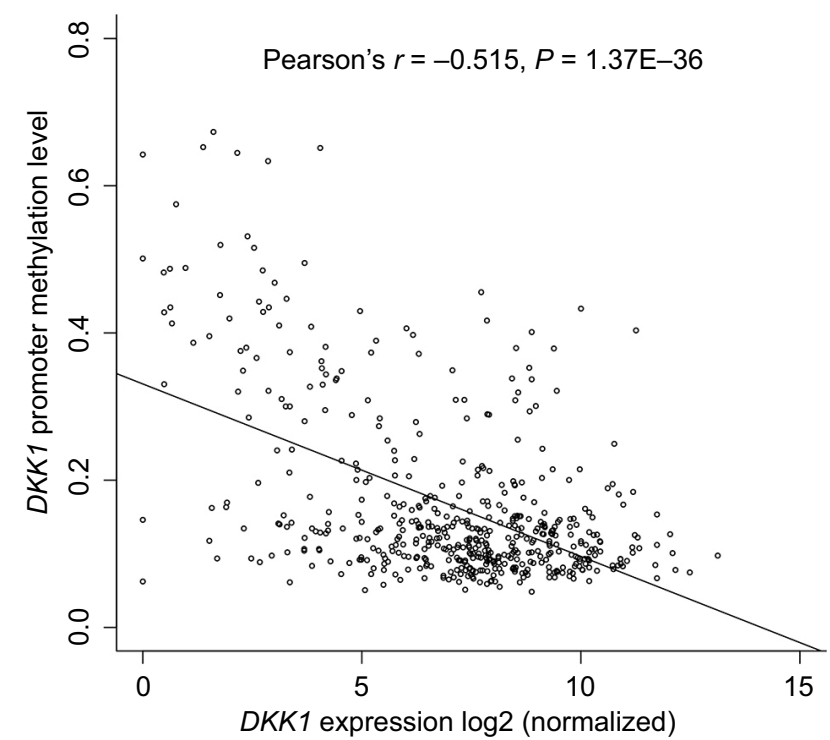

Figure 3 The correlation between DKKI expression and DNA methylation of its promoter in HNSCC tissues.

Note: DKKI expression was negatively correlated with methylation of its promoter in HNSCC.

Abbreviation: HNSCC, head and neck squamous cell carcinoma.

gene expression and modulate cancer cell behavior. As one of the most important epigenetic alterations, aberrant DNA methylation in the promoter region can directly affect gene transcription in numerous cancers, including HNSCC..$^{15,16}$ Regression analysis confirmed a significant negative correlation between DKK1 expression and methylation of its promoter, suggesting that DNA methylation may be an important mechanism influencing $D K K 1$ expression in HNSCC.

\section{Conclusion}

In the current study, we took advantage of public data and identified that high $D K K 1$ expression may be mediated by methylation of its promoter and that this gene is a highly informative prognostic biomarker for patients with HNSCC. However, a well-designed prospective large sample size study and further cell experiments are urgently needed to ascertain our results.

\section{Acknowledgment}

This research was supported by grants from the National Natural Science Foundation of China (No. 81670920 and No. 81541039), Natural Science Foundation of Zhejiang Province (No. LY15H130003), Medical and Health Science Research Foundation of Zhejiang Province (No. 2016KYB272), Huimin Technology Research and Development Projects of
Ningbo (No. 2015C50026), and Ningbo Health Branding Subject Fund (PPXK2018-02).

\section{Disclosure}

The authors report no conflicts of interest in this work.

\section{References}

1. Vigneswaran N, Williams MD. Epidemiologic trends in head and neck cancer and aids in diagnosis. Oral Maxillofac Surg Clin North Am. 2014;26(2):123-141.

2. Siegel RL, Miller KD, Jemal A. Cancer statistics, 2018. CA Cancer J Clin. 2018;68(1):7-30.

3. Siegel RL, Miller KD, Jemal A. Cancer statistics, 2017. CA: A Cancer J Clin. 2017;67(1):7-30.

4. Shield KD, Ferlay J, Jemal A, et al. The global incidence of lip, oral cavity, and pharyngeal cancers by subsite in 2012. CA Cancer J Clin. 2017;67(1):51-64.

5. Seiwert TY, Cohen EE. State-of-the-art management of locally advanced head and neck cancer. Br J Cancer. 2005;92(8):1341-1348.

6. Marur S, Forastiere AA. Head and Neck Squamous Cell Carcinoma: Update on Epidemiology, Diagnosis, and Treatment. Mayo Clin Proc. 2016;91(3):386-396.

7. Olsen JJ, Pohl SÖ, Deshmukh A, et al. The Role of Wnt Signalling in Angiogenesis. Clin Biochem Rev. 2017;38(3):131-142.

8. Anastas JN, Moon RT. WNT signalling pathways as therapeutic targets in cancer. Nat Rev Cancer. 2013;13(1):11-26.

9. Choi HJ, Park H, Lee HW, Kwon YG. The Wnt pathway and the roles for its antagonists, DKKS, in angiogenesis. IUBMB Life. 2012;64(9):724-731.

10. Hirata $\mathrm{H}$, Hinoda $\mathrm{Y}$, Nakajima K, et al. Wnt antagonist DKK1 acts as a tumor suppressor gene that induces apoptosis and inhibits proliferation in human renal cell carcinoma. Int J Cancer. 2011;128(8):1793-1803.

11. Pang H, Ma N, Shen W, et al. Effects of DKK1 overexpression on bone metastasis of SBC-3 cells. Oncol Lett. 2018;15(5):6739-6744.

12. Hall CL, Daignault SD, Shah RB, Pienta KJ, Keller ET. Dickkopf-1 expression increases early in prostate cancer development and decreases during progression from primary tumor to metastasis. Prostate. 2008;68(13):1396-1404.

13. Shi Y, Gong HL, Zhou L, Tian J, Wang Y. Dickkopf-1 is a novel prognostic biomarker for laryngeal squamous cell carcinoma. Acta Otolaryngol. 2014;134(7):753-759.

14. Cancer Genome Atlas Research Network, Weinstein JN, Collisson EA, et al. The Cancer Genome Atlas Pan-Cancer analysis project. Nat Genet. 2013;45(10):1113-1120.

15. Zhou C, Li J, Li Q. CDKN2A methylation in esophageal cancer: a meta-analysis. Oncotarget. 2017;8(30):50071-50083.

16. Shen Z, Zhou C, Li J, et al. SHISA3 Promoter Methylation Is a Potential Diagnostic and Prognostic Biomarker for Laryngeal Squamous Cell Carcinoma. Biomed Res Int. 2017;2017:9058749.

17. Lin B, Zhou X, Lin S, et al. Epigenetic silencing of PRSS3 provides growth and metastasis advantage for human hepatocellular carcinoma. J Mol Med (Berl). 2017;95(11):1237-1249.

18. Liang L, He H, Lv R, et al. Preliminary mechanism on the methylation modification of Dkk-1 and Dkk-3 in hepatocellular carcinoma. Tumour Biol. 2015;36(2):1245-1250.

19. Na Y, Lee SM, Kim DS, Park JY. Promoter methylation of Wnt antagonist DKK1 gene and prognostic value in Korean patients with non-small cell lung cancers. Cancer Biomark. 2012;12(2):73-79.

20. Galamb O, Kalmár A, Péterfia B, et al. Aberrant DNA methylation of WNT pathway genes in the development and progression of CIMPnegative colorectal cancer. Epigenetics. 2016;11(8):588-602.

21. Paluszczak J, Sarbak J, Kostrzewska-Poczekaj M, et al. The negative regulators of Wnt pathway-DACH1, DKK1, and WIF1 are methylated in oral and oropharyngeal cancer and WIF1 methylation predicts shorter survival. Tumour Biol. 2015;36(4):2855-2861. 
22. Luo J, Chen M, Liu Y, et al. Nature-derived lignan compound VB-1 exerts hair growth-promoting effects by augmenting Wnt/ $\beta$-catenin signaling in human dermal papilla cells. PeerJ. 2018;6:e4737.

23. Hong SA, Yoo SH, Lee HH, et al. Prognostic value of Dickkopf-1 and $\beta$-catenin expression in advanced gastric cancer. BMC Cancer. 2018;18(1):506.

24. Fatima S, Luk JM, Poon RT, Lee NP. Dysregulated expression of dickkopfs for potential detection of hepatocellular carcinoma. Expert Rev Mol Diagn. 2014;14(5):535-548.

25. Blot WJ, McLaughlin JK, Winn DM, et al. Smoking and drinking in relation to oral and pharyngeal cancer. Cancer Res. 1988;48(11):3282-3287.

26. Spence T, Bruce J, Yip KW, Liu FF. HPV Associated Head and Neck Cancer. Cancers (Basel). 2016;8(8):75.

27. Joseph AW, D'Souza G. Epidemiology of human papillomavirus-related head and neck cancer. Otolaryngol Clin North Am. 2012;45(4):739-764.

28. Parfenov M, Pedamallu CS, Gehlenborg N, et al; Cancer Genome Atlas Network. Characterization of HPV and host genome interactions in primary head and neck cancers. Proc Natl Acad Sci USA. 2014;111(43): 15544-15549.

29. Talmi YP, Takes RP, Alon EE, et al. Prognostic value of lymph node ratio in head and neck squamous cell carcinoma. Head Neck. 2018;40(5):1082-1090.

30. Huang SH, Xu W, Waldron J, et al. Refining American Joint Committee on Cancer/Union for International Cancer Control TNM stage and prognostic groups for human papillomavirus-related oropharyngeal carcinomas. J Clin Oncol. 2015;33(8):836-845.
31. Feng M, Wang W, Fan Z, et al. Tumor volume is an independent prognostic indicator of local control in nasopharyngeal carcinoma patients treated with intensity-modulated radiotherapy. Radiat Oncol. 2013;8:208.

32. Cooper JS, Pajak TF, Forastiere AA, et al; Radiation Therapy Oncology Group 9501/Intergroup. Postoperative concurrent radiotherapy and chemotherapy for high-risk squamous-cell carcinoma of the head and neck. N Engl J Med. 2004;350(19):1937-1944.

33. Schlitter AM, Jesinghaus M, Jäger C, et al. pT but not $\mathrm{pN}$ stage of the 8th TNM classification significantly improves prognostication in pancreatic ductal adenocarcinoma. Eur J Cancer. 2017;84:121-129.

34. Chen K, Chen H, Yang F, Sui X, Li X, Wang J. Validation of the Eighth Edition of the TNM Staging System for Lung Cancer in 2043 Surgically Treated Patients With Non-small-cell Lung Cancer. Clin Lung Cancer. 2017;18(6):e457-e466.

35. Chen C, Zhou H, Zhang X, Ma X, Liu Z, Liu X. Elevated levels of Dickkopf-1 are associated with $\beta$-catenin accumulation and poor prognosis in patients with chondrosarcoma. PLoS One. 2014;9(8):e105414.

36. Sun DK, Wang L, Wang JM, Zhang P. Serum Dickkopf-1 levels as a clinical and prognostic factor in patients with bladder cancer. Genet Mol Res. 2015;14(4):18181-18187.

37. Han SX, Zhou X, Sui X, et al. Serum dickkopf-1 is a novel serological biomarker for the diagnosis and prognosis of pancreatic cancer. Oncotarget. 2015;6(23):19907-19917.
Cancer Management and Research

\section{Publish your work in this journal}

Cancer Management and Research is an international, peer-reviewed open access journal focusing on cancer research and the optimal use of preventative and integrated treatment interventions to achieve improved outcomes, enhanced survival and quality of life for the cancer patient The manuscript management system is completely online and includes

\section{Dovepress}

a very quick and fair peer-review system, which is all easy to use. Visit http://www.dovepress.com/testimonials.php to read real quotes from published authors. 